\title{
Fabrication and cold test of dielectric assist accelerating structure
}

\author{
D. Satoh, ${ }^{1, *}$ M. Yoshida, ${ }^{1}$ and N. Hayashizaki ${ }^{2}$ \\ ${ }^{1}$ High Energy Accelerator Research Organization KEK, 1-1 Oho, Tsukuba, Ibaraki 305-0801, Japan \\ ${ }^{2}$ Laboratory for Advanced Nuclear Energy, Tokyo Institute of Technology, \\ 2-12-1 Ookayama, Meguro, Tokyo 152-8550, Japan \\ (Received 26 May 2017; published 12 September 2017)
}

\begin{abstract}
We present the detailed description of a successful design and cold testing of the dielectric assist accelerating (DAA) structure. The DAA structure consists of ultralow-loss dielectric cylinders and disks with irises which are periodically arranged in a metallic enclosure. The advantage of the DAA structure is that it has an extremely high quality factor and a very high shunt impedance at room temperature since the electromagnetic field distribution of accelerating mode can be controlled by dielectric parts so that the wall loss on the metallic surface is greatly reduced. A prototype of the five-cell DAA structure was designed and built at C-band (5.712 GHz), and cold tested. Three types of dielectric cell structure, "regular," "end," and "hybrid" dielectric cells, are fabricated by sintering high-purity magnesia. The prototype was assembled by stacking these cells in the hollow copper cylinder, whose two ends are closed by copper plates. The resonant frequency of the prototype was tuned to the desired frequency by machining only end copper plates. The unloaded quality factor of the accelerating mode was measured at 119,314 and the shunt impedance per unit length of the prototype was estimated from the experimental results of the bead pull measurement as $Z_{\mathrm{sh}}=617 \mathrm{M} \Omega / \mathrm{m}$, which were within 2 percent of the design values. The field distribution of accelerating mode was also measured by the bead pull method, and its results agreed well with simulation results.
\end{abstract}

DOI: 10.1103/PhysRevAccelBeams.20.091302

\section{INTRODUCTION}

Room-temperature radio-frequency (rf) linac structures are widely used in industrial and medical applications, such as material and radiation processing [1,2], cancer therapy [3], or imaging [4], as well as the basic research such as synchrotron radiation-based science [5] and particle colliders [6,7]. In future linear accelerators, such as an energy frontier electron positron collider, higher gradient and higher power efficiency accelerating structures are required towards compact and cost-effective devices for accelerating beams to extremely high energies.

Over the past several decades, disk-loaded copper structures have been a subject of much research. Recently, several groups demonstrated a high accelerating gradient of $100-150 \mathrm{MV} / \mathrm{m}$ in both traveling-wave and standing-wave cavities at X-band frequency with rf pulses of 100s ns [8-10]. Much higher accelerating gradients, up to $300 \mathrm{MV} / \mathrm{m}$ were obtained at $115-140 \mathrm{GHz}$, though with very short rf pulses of a few ns [11]. However, compared to superconducting cavities which realize a much higher quality factor $\left(Q_{0}\right)$ and a higher shunt impedance $\left(Z_{\mathrm{sh}}\right)$,

\footnotetext{
*daisuke.satoh@kek.jp
}

Published by the American Physical Society under the terms of the Creative Commons Attribution 4.0 International license. Further distribution of this work must maintain attribution to the author(s) and the published article's title, journal citation, and DOI. normal-conducting rf linac structures are substantially less advantageous in terms of the power efficiency. A roomtemperature rf linac structure with $Q_{0}$ and $Z_{\mathrm{sh}}$ much higher than those of present conventional normal-conducting rf linac structure, if realized, would put forward a major breakthrough for future accelerator. Thus, there still is notable room for technological innovation of roomtemperature operating rf accelerating structures.

A potential alternative to conventional disk-loaded copper structure is dielectric based accelerating structures. The most popular structure among them is a dielectric loaded accelerating (DLA) structure [12-14], which comprises a simple geometry where a dielectric tube is surrounded by a conducting cylinder. The simplicity of the DLA structure offers a great advantage for high frequency ( $>\mathrm{X}$-band) accelerating structures as compared with conventional metal structures which demand extremely tight fabrication tolerances [12]. Another advantage of DLA is that the beam-induced deflection modes are easily damped with a simple structure design [15].

Since the basic theory of DLA structures were first proposed in the 1940s [16-18], numerous studies have examined the use of dielectric materials in accelerating structures [19-27]. Recently, new concepts of dielectric based accelerating structures with improved performance have come along, such as a dual-layered DLA structure [21], a hybrid dielectric and iris loaded periodic accelerating 
structure [22], a multicell disk-and-ring tapered structure $[23,24]$, an over-mode hybrid photonic band gap accelerating structure [25], and a multilayered DLA structure $[26,27]$ which is operated by a higher order mode. Thus, the possibilities of dielectric based accelerating structures are gradually expanding. In addition, they are presently drawing renewed attention due to remarkable progress in new ceramic materials with high dielectric permittivity $\left(\varepsilon_{r}>20\right)$ and low loss $\left(\tan \delta<10^{-4}\right)$ [28-30], and, more recently, in ultralow-loss types [31-33]. However, at present the conventional copper structures are still superior in the power efficiency and the highest accelerating gradient.

Among many dielectric based accelerating structures, a dielectric assist accelerating (DAA) structure [34] appears to be very promising in terms of power efficiency. A conceptual illustration of a multicell DAA structure is shown in Fig. 1(a). It is a higher-order $\mathrm{TM}_{02 n}$ mode standing-wave accelerating structure, which consists of ultralow-loss dielectric cylinders and disks with irises which are periodically arranged in a metallic enclosure. The advantage of DAA structure is that it has an extremely high quality factor and a high shunt impedance at room temperature since the electromagnetic field distribution of the accelerating mode can be controlled by dielectric parts which greatly reduces the wall loss on metallic surface [34]. A simulation result of a five-cell DAA structure with an existing alumina ceramic indicated an unloaded $Q$ factor of the accelerating mode over 120,000 and a shunt impedance exceeding $650 \mathrm{M} \Omega / \mathrm{m}$ at room temperature [34].

(a)

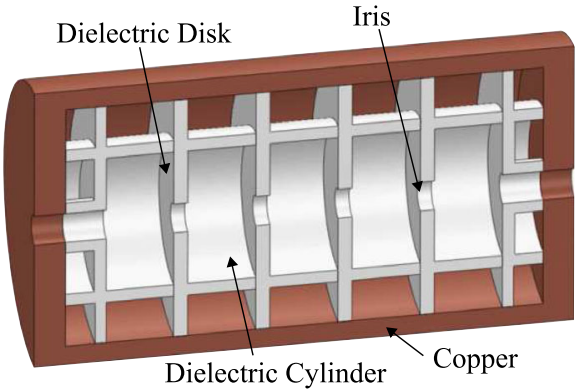

(b)

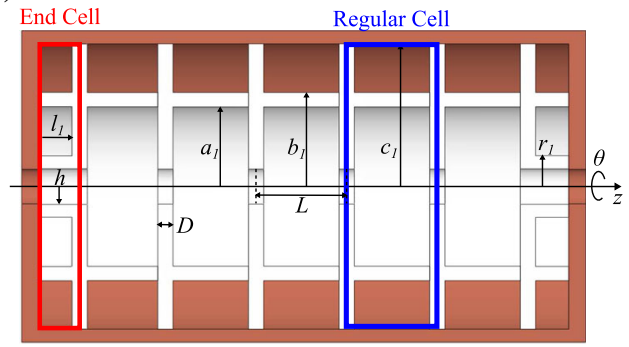

FIG. 1. (a) Conceptual illustration of a multicell DAA structure. (b) Longitudinal cross section of the DAA structure. Blue and red areas represent the regular cell and the end cell, respectively.
This paper provides a detailed description of the design, fabrication, and cold tests of a series of multicell DAA structures. The paper is organized as follows. In Sec. II, we present the rf design of a five-cell DAA structure, including frequency tuning structure. In Sec. III, we describe the fabrication and preliminary cold tests of the DAA structure, where its resonant characteristics were measured by using an electric field coupling monopole antenna. Section IV presents the rf design of an input rf coupler and cold tests of a complete DAA structure including the coupler and the mode convertor. Section VI gives the conclusions. Results from the high power test that is currently in progress will be reported in a separate publication.

\section{DESIGN OF DIELECTRIC ASSIST ACCELERATING STRUCTURE}

\section{A. rf design of DAA structure}

The DAA structure comprises two types of cell structures: a regular cell and an end cell. The areas enclosed by blue and red lines in Fig. 1(b) indicate a regular cell and an end cell, respectively. The regular cells provide the beam with accelerating field. Therefore, the design of the regular cell plays a dominant role in determining the accelerator parameters such as the quality factor and the shunt impedance, while the end cells, arranged at the two ends of the DAA structure, are provided to reduce the $\mathrm{rf}$ dissipation on the surface of both conducting end plates and they do not contribute to beam acceleration.

In the following, this paper deals with a "five-cell" C-band DAA standing-wave structure which was designed as a prototype model, consisting of five regular cells and two end cells, to operate at the C-band $(5.712 \mathrm{GHz})$ frequencies in the $\pi$ mode. With considerations on the dielectric properties (e.g., a relative permittivity $\varepsilon_{r}$ and loss tangent $\tan \delta$ ), production cost, and mass productiveness of ceramics, we selected polycrystalline magnesia as the dielectric cell material. The magnesia ceramic which was used for the prototype model has the purity of $3 \mathrm{~N}$ class. From the resonant characteristics of a dielectric resonator made with the same material, we have estimated its relative permittivity $\varepsilon_{r}$ and $\tan \delta$ near $10 \mathrm{GHz}$ as 9.64 and $6.0 \times 10^{-6}$ at room temperature, respectively [35]. The geometric parameters were optimized by a down-hill simplex algorithm [36] to maximize the shunt impedance per unit length $Z_{\mathrm{sh}}$ of the prototype. A design procedure of the prototype is detailed in Appendix A. Table I presents the computed accelerator characteristics of the designed prototype, including the unloaded quality factor, $Q_{0}$, and the shunt impedance per unit length, $Z_{\mathrm{sh}}$. Here, the $E_{0}, E_{\max }$, and $H_{\text {max }}$ represent an average accelerating gradient, a peak surface electric field, and a peak surface magnetic field, respectively. 
TABLE I. Basic cavity and accelerator parameters of an optimized five-cell DAA structure.

\begin{tabular}{lc}
\hline \hline Parameter & Five-cell DAA structure \\
\hline Dielectric material & Magnesia \\
$\varepsilon_{r}$ & 9.64 \\
$\tan \delta$ & $6.0 \times 10^{-6}$ \\
Accelerator type & Standing wave type \\
Accelerating mode & $\mathrm{TM}_{02}-\pi$ mode \\
Operation frequency & $5.712 \mathrm{GHz}$ \\
Number of accelerating cells & 5 \\
Total cavity length & $157.5 \mathrm{~mm}$ \\
$Q_{0}$ & 126,400 \\
$Z_{\mathrm{sh}}$ & $630 \mathrm{M} \Omega / \mathrm{m}$ \\
$E_{\max } / E_{0}$ & 2.92 \\
$H_{\max } / E_{0}$ & $2.74 \mathrm{~mA} / \mathrm{V}$ \\
\hline \hline
\end{tabular}

\section{B. Frequency tuning structure}

The resonant frequency of DAA structure could be tuned by machining a concentric circular groove on the surface of the end copper plates. Figure 2 shows the conceptual illustrations of the end cell containing a frequency tuning structure. The inner and outer radii of the concentric circular groove are set to $r_{1}$ and $a_{1}$, respectively. The depth of the groove is defined as $d$. Shifts of the resonant frequency $\Delta f_{0}$ and shunt impedance $\Delta Z_{\mathrm{sh}}$ as functions of the groove depth $d$ is shown in Fig. 3. The resonant frequency of the prototype can be tuned down by up to about $30 \mathrm{MHz}$ if the grooves are made $4 \mathrm{~mm}$ depth. In addition to the effect of frequency tuning, the shunt impedance $Z_{\mathrm{sh}}$ can be improved by up to about 2.2 percent. Figure 4 shows the normalized longitudinal electric field strengths on the beam axis in the prototype models with $d=0.0 \mathrm{~mm}$ and $d=4.0 \mathrm{~mm}$. It indicates that while the peak strengths of the accelerating field in the first and the fifth regular cells is decreased by 5.8 percent, the distribution of the on-axis accelerating field as a whole is hardly influenced by this tuning. Taking all these calculations

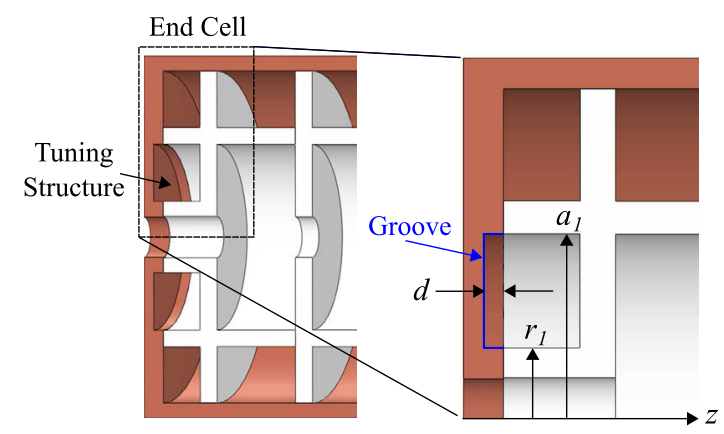

FIG. 2. Conceptual illustrations of the end cell containing a frequency tuning structure. The inner and outer radii of the concentric circular groove are set to $r_{1}$ and $a_{1}$, respectively. The groove depth of it is defined in $d$.

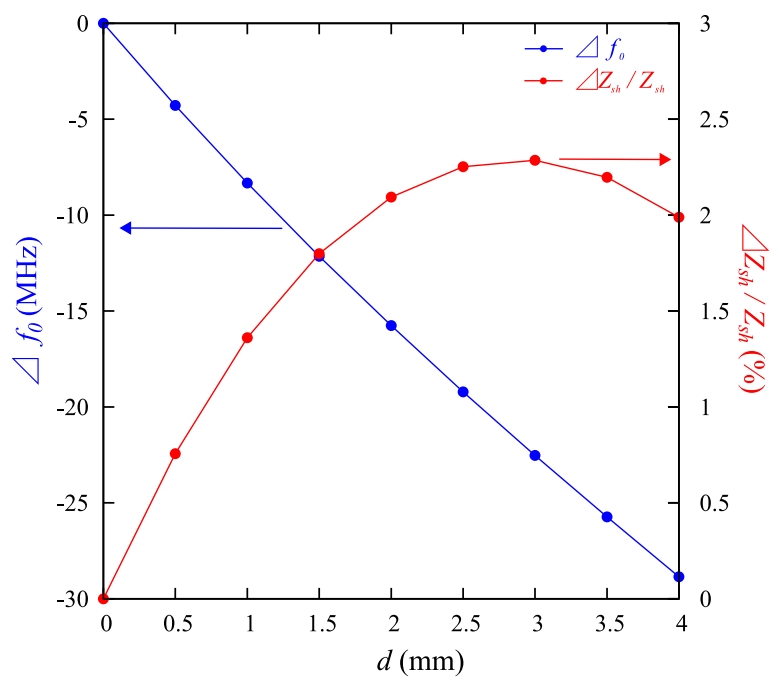

FIG. 3. Shifts of the resonant frequency $\Delta f_{0}$ and shunt impedance $\Delta Z_{\mathrm{sh}}$ as functions of the groove depth $d$. The shifts of resonant frequency are defined as $\Delta f_{0}=f_{0}(d)-f_{0}(0)$. The shifts of shunt impedance are defined as $\Delta Z_{\mathrm{sh}} / Z_{\mathrm{sh}}=$ $\left\{Z_{\mathrm{sh}}(d)-Z_{\mathrm{sh}}(0)\right\} / Z_{\mathrm{sh}}(0)$.

into consideration, the preferred construction scenario of this structure is to fabricate the dielectric cells for frequencies slightly higher than the design frequency and compensate it with appropriate tuning on the end copper plates. The tolerances of geometrical parameters were also studied and some representative results were described in Appendix B.

In addition to designing the prototype of the DAA structure, an input coupler must also be designed separately. The input coupler was designed with inputs obtained from the preliminary cold tests of the fabricated prototype using an antenna feeder. The detailed design of the input coupler was described in Sec. IV.

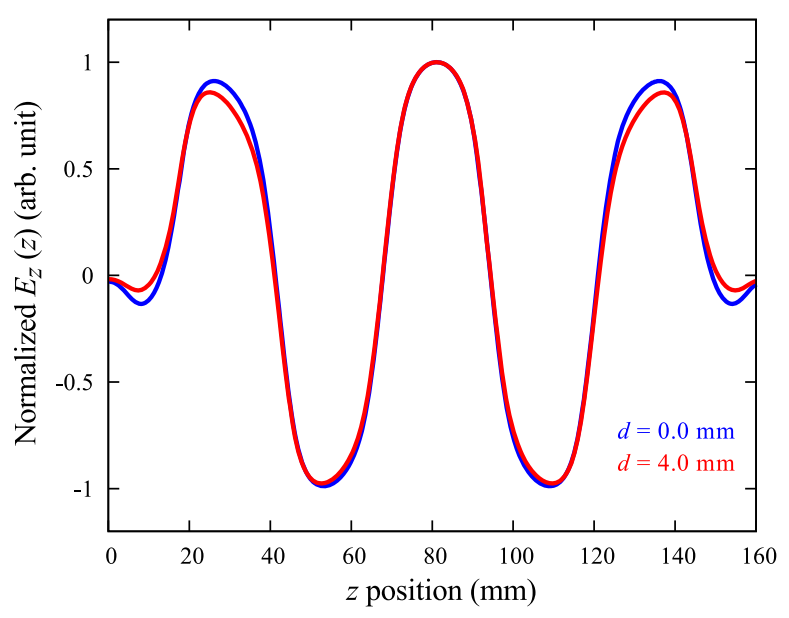

FIG. 4. Normalized longitudinal electric field strengths on the beam axis in the prototype models with $d=0.0 \mathrm{~mm}$ (blue line) and $d=4.0 \mathrm{~mm}$ (red line). 


\section{FABRICATION AND PRELIMINARY COLD TESTS OF THE DAA STRUCTURE}

\section{A. Fabrication of the DAA structure}

In order to experimentally verify the extremely high quality factor and a very high shunt impedance of the DAA structure at room temperature, we developed a prototype structure. Metallic parts of the prototype were fabricated using an oxygen-free-high-conductivity (OFHC) copper hollow cylinder closed at each end by OFHC copper circular plates. For the dielectric cell structures, we fabricated three types of specially designed structures in which the dielectric cylinders were integrally formed on the surface of a dielectric disk in a vertical direction. The fabricated dielectric cell structures are shown in Fig. 5. The dielectric
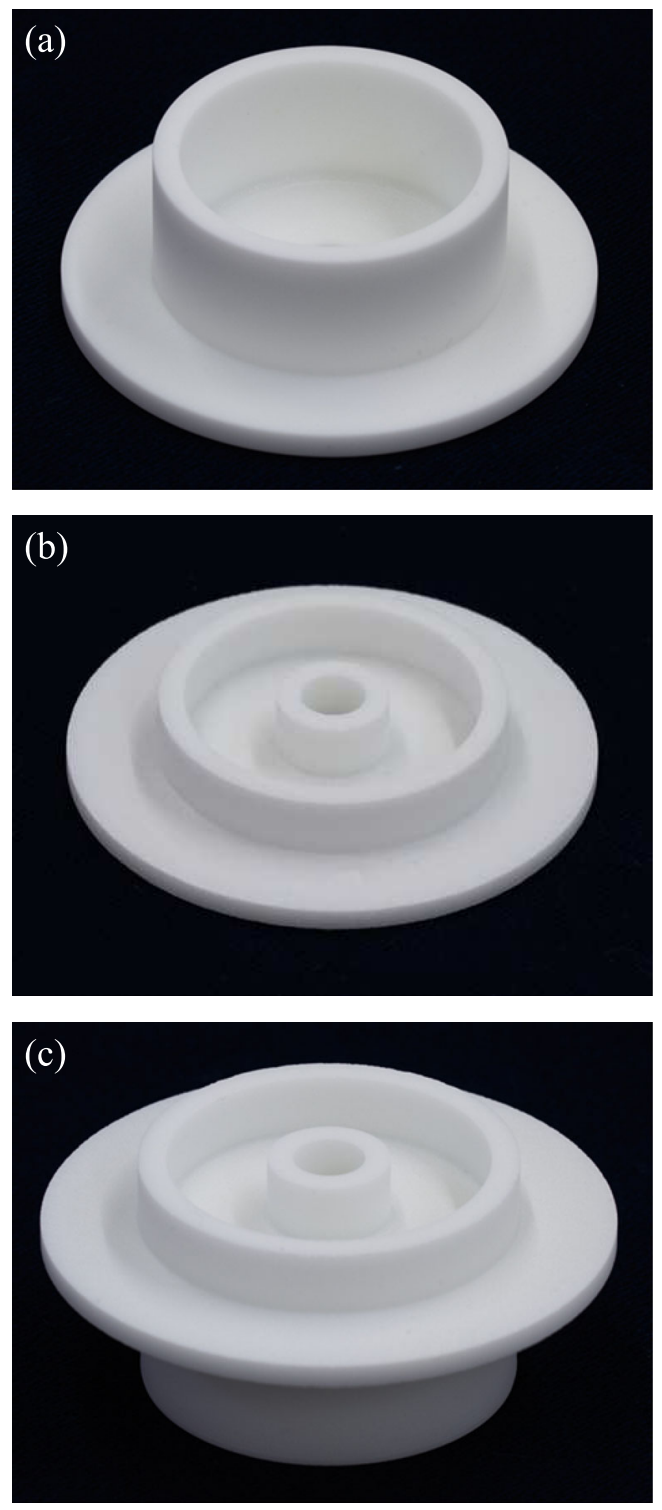

FIG. 5. The fabricated dielectric cell structures: (a) regular dielectric cell, (b) end dielectric cell, (c) hybrid dielectric cell. cell structures shown in Figs. 5(a) and 5(b) correspond to a regular dielectric cell and an end dielectric cell, respectively. The dielectric cell structure shown in Fig. 5(c) is a hybrid dielectric cell, which has the regular and the end dielectric cells integrally formed through a dielectric disk. These dielectric cell structures were fabricated as follows: First, a fine magnesia powder was combined and kneaded with a ceramic binder. Next, the granulated powder was filled in a metal mold die, and columnar raw ceramic compacts were produced by a pressure molding method. Then these compacts were machined by a lathe into appropriate shapes while taking into account the shrinkage factors during sintering, and the machined ones were sintered at a temperature over $2000 \mathrm{~K}$. Then the prototype structure was assembled by stacking the end dielectric cell, the four regular dielectric cells, and the hybrid dielectric cell in the hollow copper cylinder.

For high power testing of the prototype, its whole interior must be evacuated into an ultrahigh vacuum. A space between the dielectric layer and metallic enclosure, however, can hardly be evacuated from a central part of it. Therefore, a number of vacuum holes (Figs. 10 and 11) are introduced on the copper cylinder and copper circular plates, rather than on dielectric ceramic cells. The sizes of the holes are chosen to be small enough so as not to leak the rf power while securing sufficient vacuum conductance. Then the prototype would be placed in a vacuum chamber, and its interior would be evacuated from these holes.

\section{B. Preliminary cold tests of DAA structure}

The dielectric cells, after fabrication and sintering, were geometrically measured and the cell-to-cell variations of their dimensional parameters were found to stay within $\pm 0.1 \mathrm{~mm}$. However, with respect to design values, some of the measured geometrical parameters exhibited systematic errors of $0.3 \mathrm{~mm}$ or more. Calculations have shown that the prototype to be assembled with these cells, as they are fabricated, will have the resonant frequency of $5.7264 \mathrm{GHz}$, approximately $14 \mathrm{MHz}$ higher than the operating frequency of $5.712 \mathrm{GHz}$.

In the preliminary cold tests of the prototype, rf power was fed and detected by an electric field coupling monopole antenna. This antenna feeder was inserted through a beam hole in parallel to the beam axis. We measured the $S_{11}$ (reflection) elements of the scattering matrix by using a vector network analyzer (VNA) while the coupling coefficient $\beta$ between the DAA structure and antenna feeder was adjusted by changing the insertion depth on the antenna feeder. The rf coupling between the prototype and the antenna was always kept to be undercoupling so as to prevent changes of the resonant characteristics. Figure 6(a) shows the frequency dependence of the measured $\left|S_{11}\right|$ on the frequency from $5.729 \mathrm{GHz}$ up to $5.731 \mathrm{GHz}$. Figure 6(b) shows the Smith chart for the measured $S_{11}$. The coupling coefficient $\beta$ was approximately 
(a)

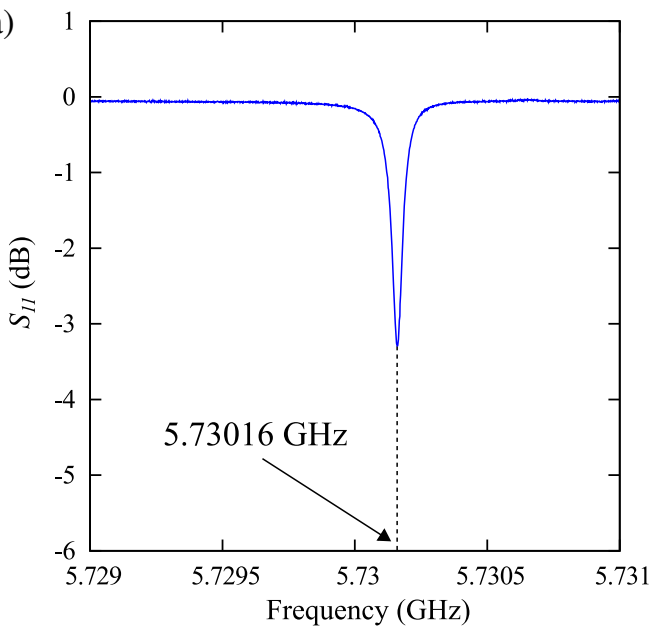

(b)

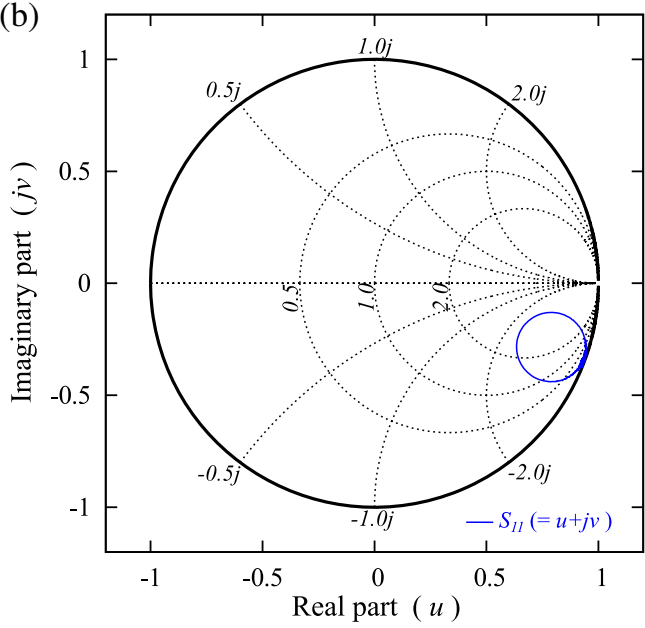

FIG. 6. (a) Frequency dependence of the measured $\left|S_{11}\right|$. The horizontal axis indicates the frequency $(\mathrm{GHz})$ and the vertical axis indicates the $S_{11}$ in decibel units $(\mathrm{dB})$. (b) The measured $S_{11}$ in the format of the Smith chart. The coupling coefficient $\beta$ was approximately 0.19 during these measurements.

0.19 during these measurements. The loaded and unloaded $Q$ factors of the DAA structure are calculated from the $S_{11}$ curve and Smith chart. Since this structure is always undercoupled with the antenna feeder present, the coupling coefficient $\beta$ is related to the minimum $S_{11}$ value, $S_{11, \min }$, as $\beta=\left(1-S_{11, \min }\right) /\left(1+S_{11, \min }\right)$. The loaded $Q$ factor $Q_{L}=$ $f / \Delta f$ can be determined from the frequency bandwidth. The unloaded $Q$ factor is $Q_{0}=(1+\beta) Q_{L} \cdot Q$ factors were also calculated by Ginzton's impedance method [37] for comparison. By using this method, the loaded $Q$ factor, the unloaded $Q$ factor and the external $Q$ factor can be independently calculated from the input impedance plotted on the Smith chart [37]. A more detailed description can be found in Ref. [37].

Figure 7 shows the dependence of the resonant frequency $f_{0}$ and the unloaded $Q$ factor $Q_{0}$ on the coupling coefficient $\beta$. Figure 7(a) indicates that the resonant
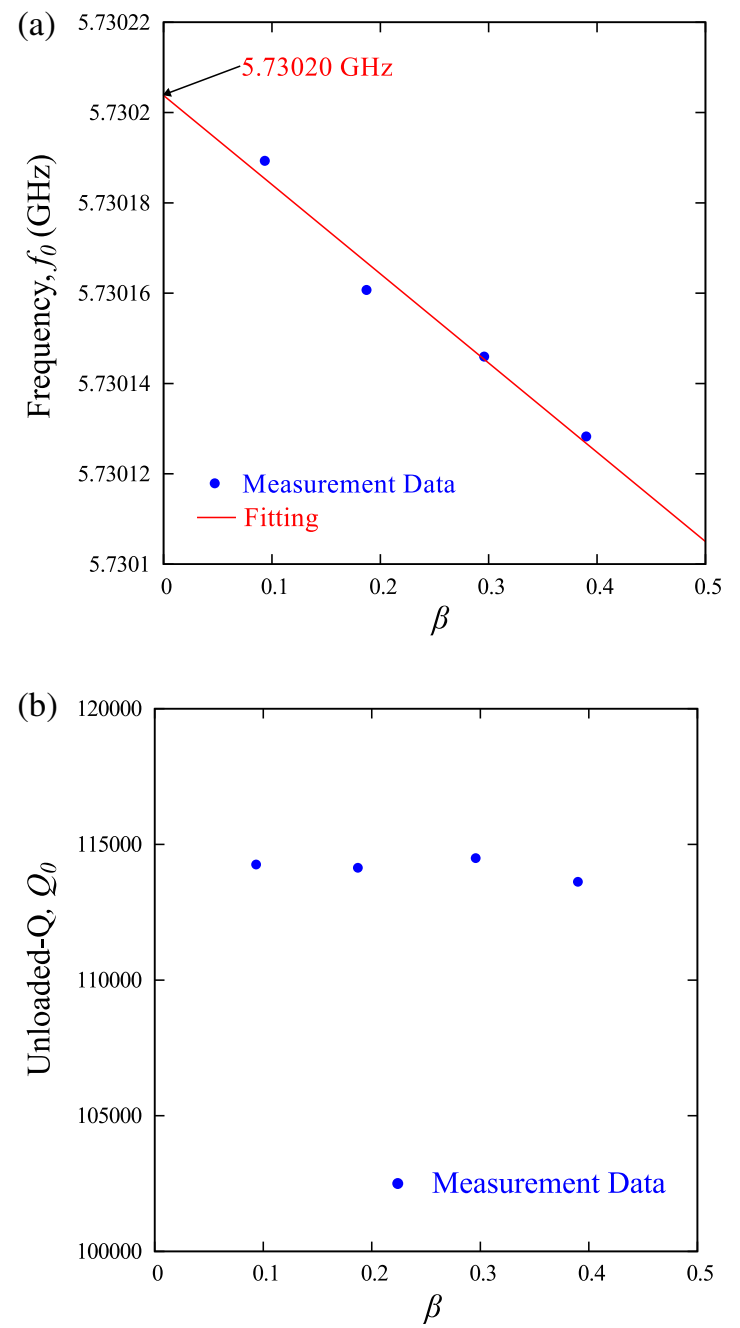

FIG. 7. Dependence of (a) the resonant frequency $f_{0}$ and (b) the unloaded $Q$ factor $Q_{0}$ on the coupling coefficient $\beta$.

frequency of the prototype is in the range of 5.73010 to $5.73022 \mathrm{GHz}$ and tends to almost shift lower in proportion to the insertion depth of the antenna. Considering an equivalent circuit of a cavity resonator, the frequency shift due to insertion of the antenna is caused by an increase of the capacitive component of the cavity. The resonant frequency of the prototype unperturbed by the insertion of the antenna, thus, is estimated with a linear function fitting of the resonant frequency $f_{0}$ as a function of the coupling coefficient $\beta$ as shown in Fig. 7(a). From the intercept of the fitting function $(\beta=0)$, the unperturbed resonant frequency of the prototype was estimated as $5.73020 \mathrm{GHz}$. This is higher (by $3.8 \mathrm{MHz}$ ) than the resonance frequency as predicted from the measured geometrical sizes of individual cells $(5.7264 \mathrm{GHz})$ as discussed earlier. The difference in these frequencies is considered attributable to some factors as follows: (i) position errors of the dielectric cells in the copper enclosure, (ii) warpage of the dielectric cells, (iii) deviations in the relative permittivity of the dielectric cells. These slight 
differences of the dielectric cells described in items (ii) and (iii) might be caused by a spatial nonuniformity of sintered density of them due to their complex structures. If the frequency difference is to be accounted for by only the effect (iii), the deviation of relative permittivity should be $0.03 \sim 0.04$. Figure 7(b) indicates that the unloaded $Q$ factor and coupling coefficient $\beta$ are not correlated and these measured values are consistent with each other within 5 percent. The average value of the measured $Q_{0}$ is 114,000 , while the simulated $Q_{0}$ considering the dimensional errors of each dielectric cell is 125,000 . It is seen that the experimentally measured $Q_{0}$ is approximately 10 percent lower than the simulated $Q_{0}$. This difference can be attributed to additional losses in the prototype structure due to imperfect electrical contact between the copper pipe and the end plates. For development of the DAA structure coupled with a waveguide input coupler, the contact portions between the copper parts were designed to be small and mirror-polished surfaces to improve the $Q_{0}$.

\section{FABRICATION AND COLD TEST OF DAA STRUCTURE WHICH IS ON-AXIS COUPLED WITH WAVEGUIDE INPUT COUPLER}

A DAA structure which is on-axis coupled with a waveguide input coupler was developed on the basis of the results of the preliminary cold tests presented in Sec. III. Figure 8 shows a conceptual illustration of the rf input coupler including a TE-TM mode converter. The rf power is transmitted from the rectangular waveguide to the circular waveguide through the TE-TM mode converter. This mode converter consists of a rectangular waveguide with a shortcircuit terminal and the circular waveguide which is perpendicular to it. There, the propagating $\mathrm{TE}_{10}$ mode in the waveguide is converted into the circular $\mathrm{TM}_{01}$ mode. Then the DAA structure is electrically coupled with this circular waveguide via an on-axis coupling slot between the waveguide and the end cell.

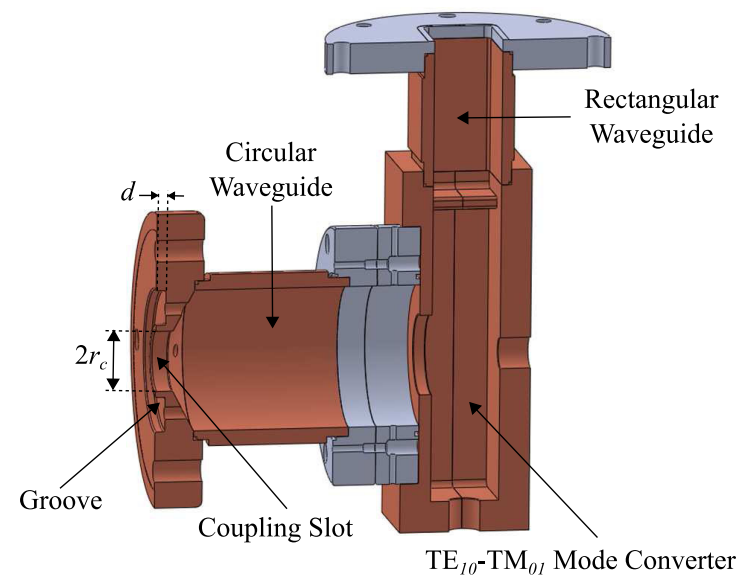

FIG. 8. Conceptual illustration of the rf input coupler including a TE-TM mode converter.
A great advantage of this input coupler design is its structural simplicity [38]. Regarding the mode converter, owing to its simplicity, the circular waveguide could be made detachable from a section of the rectangular waveguide closed by a short-circuit terminal. As a result, the coupling coefficient $\beta$ between the DAA structure and the circular waveguide can be freely tuned by changing the coupling structure.

The coupling between the DAA structure and the circular waveguide was calculated by using the Slater's tuning curve method [39] with SUPERFISH code [40]. First, the resonant frequencies of the one-port cavity resonator which consists of the DAA structure and the circular waveguide were calculated while changing the position of an electrical short boundary on the side of the circular waveguide. Then the resonant frequency $f_{0}$ and external $Q$ factor $Q_{\text {ext }}$ of the DAA structure were estimated from the tuning curves which were obtained from the above simulations of the one-port cavity resonator. Since the resonant frequency $f_{0}$ and the external $Q$ factor $Q_{\text {ext }}$ depend on the radius of coupling slot $r_{c}$ and the groove depth $d$, we calculated $f_{0}$ and $Q_{\text {ext }}$ for various combinations of $r_{c}$ and $d$. In the design of the input coupler, the coupling coefficient $\beta$ between the DAA structure and the circular waveguide was chosen to be about 2.5 , so that its filling time is sufficiently shorter than $10 \mu$ s which is the maximum pulse width easily attainable from the C-band klystron at KEK.

Figure 9 shows the dependence of the resonant frequency $f_{0}$ and the external $Q$ factor $Q_{\text {ext }}$ of the DAA structure on the groove depth $d$. In this case, the groove depth of the end plate opposite to the input coupler was also set at $d$. It shows that by assuming the radius of coupling slot $r_{c}$ to be $9.8 \mathrm{~mm}$ and the groove depth $d$ as $3.2 \mathrm{~mm}$, the $f_{0}$ matches with the C-band frequency $(5.712 \mathrm{GHz})$. The $Q_{\text {ext }}$ (external $Q$ factor) in this case will be 46,560 , which is equivalent to $\beta=2.45$ if the measured $Q_{0}$ is used for the

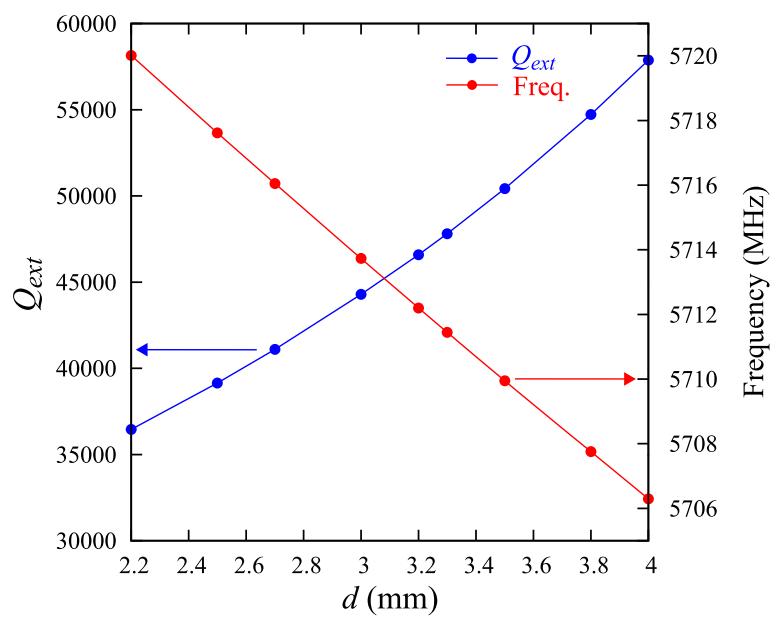

FIG. 9. Dependence of the resonant frequency $f_{0}$ (red dot line) and the external $Q$ factor $Q_{\text {ext }}$ (blue dot line) of the DAA structure on the groove depth $d$. The radius of coupling slot $r_{c}$ is $9.8 \mathrm{~mm}$. 


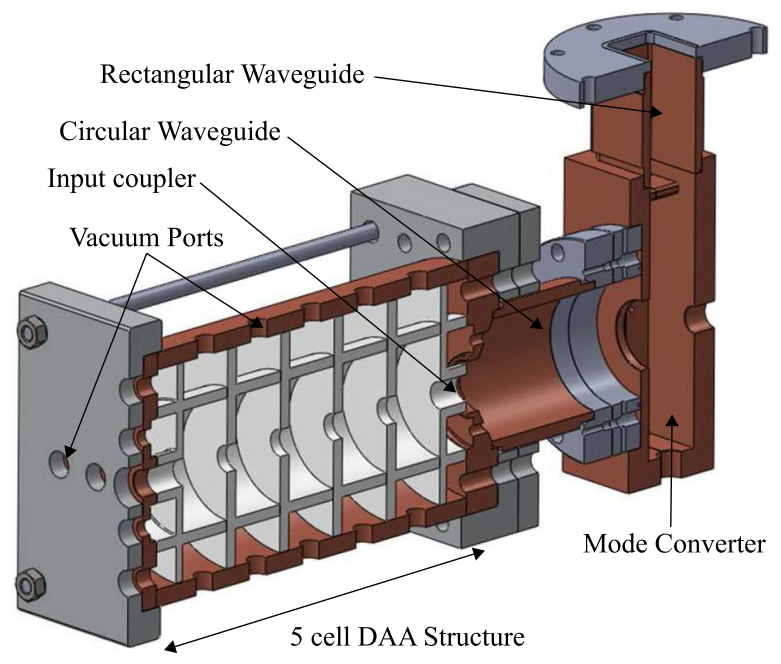

FIG. 10. Schematic half-cross section of the DAA structure assembly including the input coupler and the TE-TM mode converter.

coupling calculation. This also matches the target value. The input coupler for our prototype was fabricated with these parameters.

Figure 10 shows a schematic half-cross section of the DAA structure assembly, including the input coupler and the TE-TM mode converter. This structure was assembled by stacking in turn the end dielectric cell, the four regular dielectric cells, and the hybrid dielectric cell in the hollow copper cylinder, and one pipe end was terminated by an end plate and the other pipe end was connected to the input coupler. The contact surfaces between the copper pipe and the input coupler were mirror polished kept small sized, so that they contact firmly by pressing from both sides. The mode convertor and the rectangular waveguide were brazed together. The circular waveguide, brazed with an input rf coupling end plate and the mode convertor, were connected by a flange structure. The DAA structure and the input coupler are held by a pair of stainless plates and four stud bolts as shown in Fig. 10 (illustration) and Fig. 11 (photo).

The $S_{11}$ curves of the DAA structure on-axis coupled with a waveguide input coupler were measured by using VNA. A waveguide-to-coaxial adapter was used to attach a coaxial cable which connects the DAA structure with the VNA. The VNA was calibrated at the connection surface of the rectangular waveguide by using one-port short-shortload waveguide calibration. The dependence of the measured $S_{11}$ frequency indicated that the resonance frequency was $5.7150 \mathrm{GHz}$, which was $3 \mathrm{MHz}$ higher than C-band frequency. The Smith chart of $S_{11}$ measured by VNA indicated that the DAA structure was overcoupled. The external $Q$ factor was estimated from the measured $S_{11}$ as $Q_{\text {ext }}=42,900$, which is about 14 percent lower than the design value. This frequency shift can be tuned by changing the groove depth of only the end plate opposite to the input coupler.

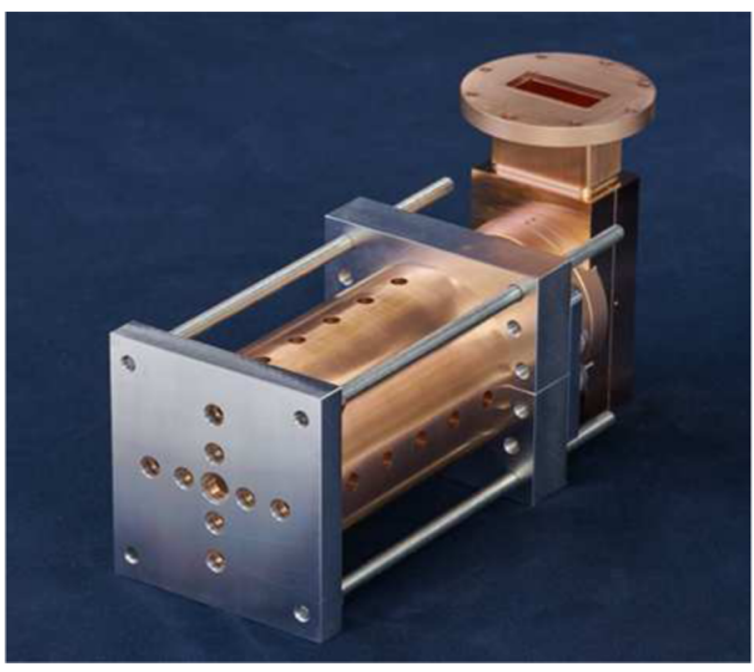

FIG. 11. Photograph of the fabricated DAA structure assembly including the input coupler and the TE-TM mode converter.

Several end plates, with different groove depths, were tried and resonant frequencies of each configuration were measured by the VNA for frequency tuning. Then the dependence of the frequency shift on the groove depth was estimated for the fine-tuning of the resonant frequency. As a result, the resonant frequency changed substantially linearly to the groove depth, so that an average change rate of the resonant frequency to the groove depth was estimated as $\Delta f / \Delta d=-3.2 \mathrm{MHz} / \mathrm{mm}$. Finally, we succeeded in tuning its resonant frequency to approximately C-band frequency by grooving a concentric circular groove with $4.2 \mathrm{~mm}$ depth $(d=4.2 \mathrm{~mm})$ on the surface of one end plate, and frequency tuning of the cold test model was finished.

The frequency-tuned DAA structure was cold-tested again to accurately estimate the $Q$ factors and the coupling. The measured $S_{11}$ curves of the frequency-tuned DAA structure were shown in Fig. 12. The comparison between the design and the measured cavity parameters of the DAA structure is shown in Table II. The measured $Q_{0}$ was in agreement, within -5.0 percent, with the simulated value. The coupling coefficient $\beta$ between the frequencytuned DAA structure and the input coupler was also in good agreement between the measured and the simulated values.

The electromagnetic field distribution of the resonant mode at about $5.712 \mathrm{GHz}$ in the DAA structure was measured by a bead pull method [41], which is based on the Slater theorem [37]. In this measurement, a small metallic or dielectric object attached to a string is pulled through the rf structure, while a small rf power is fed into the structure at the frequency of the resonant mode. The frequency shift due to the insertion of the perturbing object is measured during the passage of the bead. The relative change in resonant frequency is proportional to the relative change in stored energy and it can be expressed as Eq. (1) 

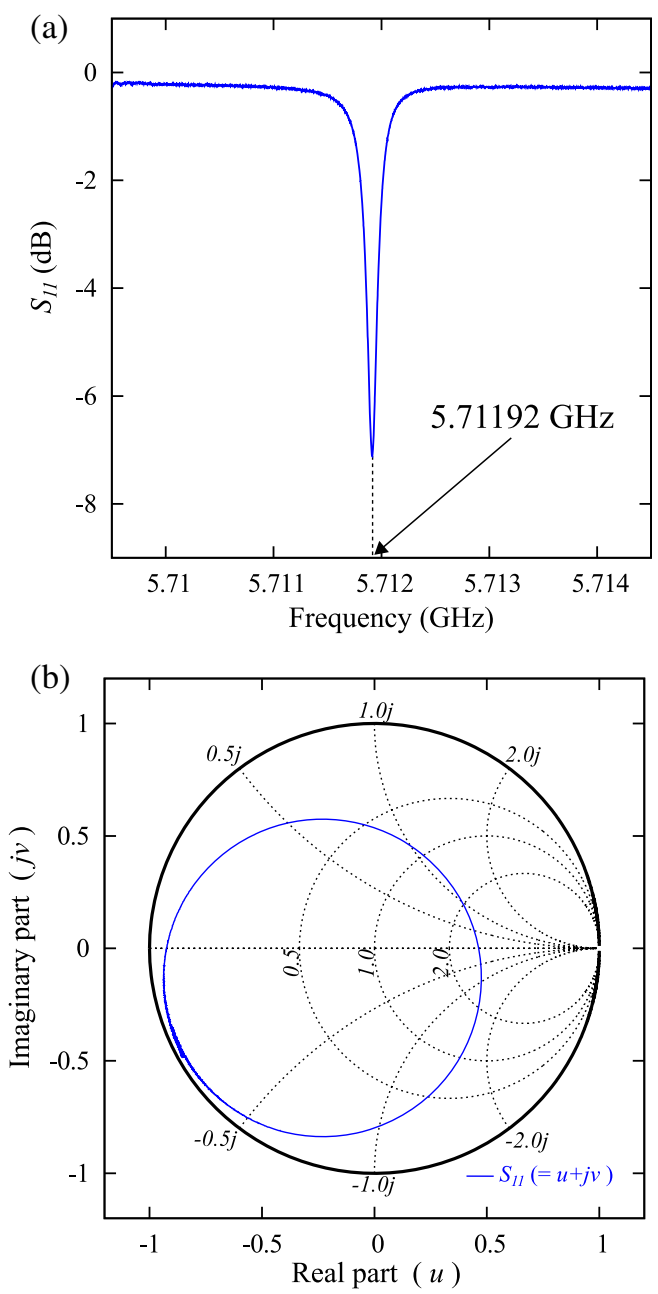

FIG. 12. (a) Frequency dependence of the measured $\left|S_{11}\right|$. The horizontal axis indicates the frequency $(\mathrm{GHz})$ and the vertical axis indicates the $S_{11}$ in decibel units (dB). (b) The measured $S_{11}$ in the format of the Smith chart.

as a function of the local electric and magnetic field strength, $E_{0}$ and $H_{0}$ [41-43]:

$$
\frac{\Delta f}{f_{0}}=F_{H} \frac{\mu_{0}\left|\vec{H}_{0}\right|^{2}}{U}-F_{E} \frac{\varepsilon_{0}\left|\vec{E}_{0}\right|^{2}}{U}
$$

TABLE II. Comparison between the design and the measured cavity parameters of the DAA structure on axis coupled with a waveguide input coupler.

\begin{tabular}{lcc}
\hline \hline Parameter & Design values & Measured values \\
\hline$f_{0}(\mathrm{GHz})$ & 5.7120 & 5.71192 \\
$Q_{0}$ & 125,000 & 119,314 \\
$Q_{\text {ext }}$ & 48,803 & 46,246 \\
$Q_{L}$ & 35,112 & 33,328 \\
$\beta$ & 2.56 & 2.58 \\
\hline \hline
\end{tabular}

where $\Delta f=f-f_{0}$, and $\varepsilon_{0}$ and $\mu_{0}$ are the dielectric and magnetic constants of the free space, $U$ is the stored energy in the structure, and $F_{E}, F_{H}$ are the geometry factors associated with the bead shape for the $\mathrm{E}$ field and $\mathrm{H}$ field. In the case of the DAA structure, the magnetic field strength of $\mathrm{TM}_{02}$ mode near the beam axis is very small $\left(\left|H_{0}\right| \sim 0\right)$. Consequently, the electric field strength of $\mathrm{TM}_{02}$ mode on the beam axis can be expressed as

$$
\left|\vec{E}_{0}(z)\right|=\sqrt{\frac{P_{\text {loss }} \cdot Q_{0}}{2 \pi \varepsilon_{0} F_{E}}\left(-\frac{\Delta f(z)}{f_{0}^{2}}\right)},
$$

where $P_{\text {loss }}$ is the total $\mathrm{rf}$ loss in the DAA structure, $z$ is the position of the bead along the beam axis. In this approximation, the electric field strength is directly proportional to the relative change in the resonant frequency.

In this measurement, a $2.0 \mathrm{~mm}$ diameter aluminum bead was mounted on a nylon wire whose diameter is $0.1 \mathrm{~mm}$. In case of a metallic sphere, the geometry factor is $F_{E}=\pi r^{3}$, with $r$ being the radius of the bead. This wire with the bead was aligned along the beam axis of the DAA structure and pulled through the beam hole with a motor. The rf power is fed through the waveguide input coupler. The VNA recorded the resonant frequency shift of the signal reflected from the structure.

Figure 13(a) compares the simulated and the measured values of $\sqrt{-\Delta f} / f_{0}$, which are expected to be in proportion with the maximum electric field on the beam axis. The simulation values were obtained by calculating the frequency shift of the accelerating mode due to the passage of the bead on the SUPERFISH code. Figure 13(b) compares the simulated longitudinal field distribution of the accelerating mode along the beam axis for the DAA structure and the experimental result of the bead pull measurement. The experimental results (blue dots) show normalized $\sqrt{-\Delta f} / f_{0}$, which are scaled by using the maximum value of $\sqrt{-\Delta f} / f_{0}$. The simulated results (red line) show normalized longitudinal electric field strength of the accelerating mode along the beam axis, which are scaled by using a maximum value of $E_{z}(z)$. Both Figs. 13(a) and 13(b) show very good agreement between the simulation and the measurement. We concluded that the resonant mode which was tuned at $5.712 \mathrm{GHz}$ is the desired accelerating mode.

The values of the $R / Q$ and shunt impedance per unit length, $Z_{\mathrm{sh}}$, of the DAA structure were also estimated from the above experimental results of the bead pull measurement. By using Eq. (2) for the accelerating field, the $R / Q$ value of the DAA structure is expressed as

$$
\begin{aligned}
\frac{R}{Q} & =\frac{\left(\int_{0}^{L}\left|\vec{E}_{0}(z)\right| d z\right)^{2}}{P_{\text {loss }} Q} \\
& =\frac{1}{f_{0}^{2}} \frac{2}{3 \pi \varepsilon_{0}} \frac{1}{\Delta V}\left(\int_{0}^{L} \sqrt{-\Delta f(z)} d z\right)^{2},
\end{aligned}
$$




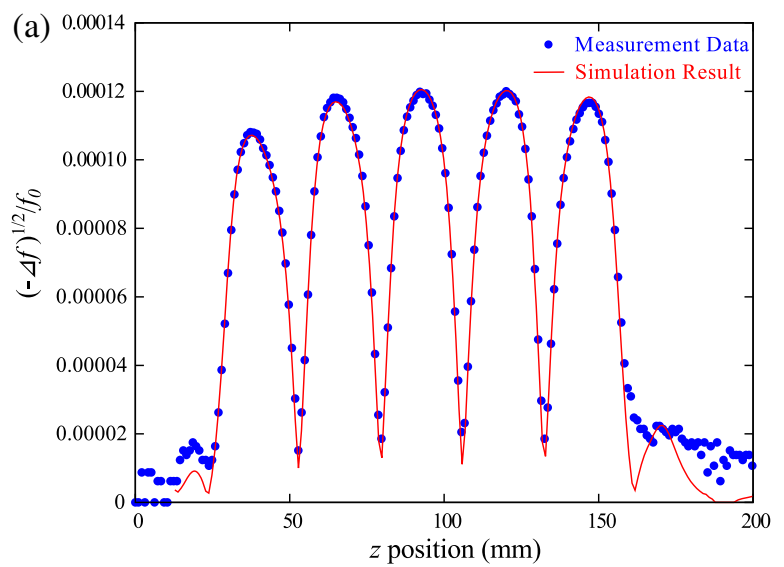

(b)

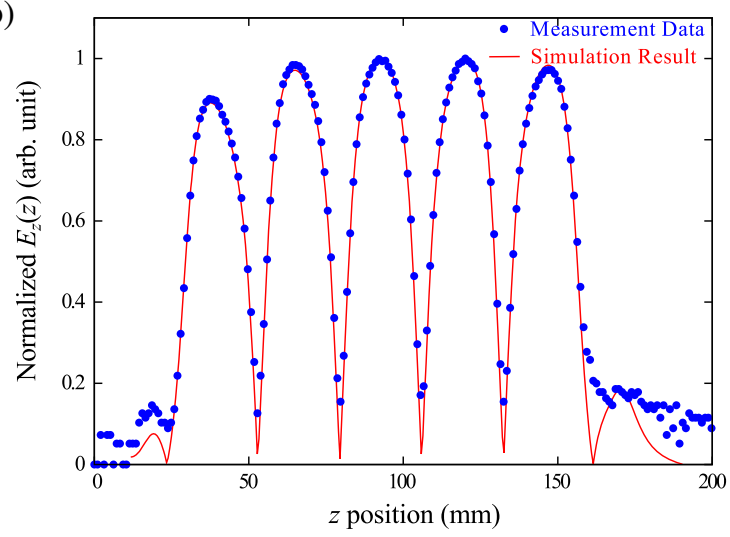

FIG. 13. (a) Comparison between the measured (blue dots) and the simulated (red line) values of $\sqrt{-\Delta f} / f_{0}$. (b) Comparison between the simulated longitudinal field distribution of the accelerating mode along the beam axis for the DAA structure and the experimental result of the bead pull measurement. The experimental results (blue dots) show normalized $\sqrt{-\Delta f} / f_{0}$, which are scaled by using the maximum value of $\sqrt{-\Delta f} / f_{0}$. The simulated results (red line) show normalized longitudinal electric field strength of the accelerating mode along the beam axis, which are scaled by using a maximum value of $E_{z}(z)$.

where $\Delta V$ is the volume of the bead, $L$ is a total length of the DAA structure. The $R / Q$ value of the DAA structure was calculated by substituting the measured $\Delta f(z)$ for Eq. (3). As a result, the $R / Q$ value was estimated as $R / Q=$ $853 \Omega$. Shunt impedance per unit length of the DAA structure was also calculated by using the estimated $R / Q$ and the measured unloaded $Q$ factor, and estimated as $Z_{\mathrm{sh}}=617 \mathrm{M} \Omega / \mathrm{m}$.

\section{DISCUSSIONS}

The preceding studies of dielectric based accelerating structures encountered dielectric joint breakdowns at microgaps [44], and rf power absorption due to single-surface multipactor when the accelerating gradient approached a few $\mathrm{MV} / \mathrm{m}[45,46]$. Charge accumulation in dielectric parts due to the beam halo has been also known [47]. These problems may occur also in the DAA structure, and if they do, they need to be addressed before the DAA structure is used in real accelerators. Thus, studies on the following subjects will have to be conducted: (1) bonding between copper and ceramic; (2) the surface coating of dielectric materials so as to reduce their secondary electron emission coefficient; (3) sintering of some ceramic materials for reasonably high dielectric permittivity $\left(\varepsilon_{r}>20\right)$ and low loss tangent $\left(\tan \delta<10^{-4}\right)$. The bonding between copper and ceramic will eliminate microgaps and improves conductive cooling of dielectric cells. Microgaps between adjacent dielectric cells may be eliminated or reduced also by positively isolating each dielectric cell. Surface coating technology will be effective in reducing the multipactor discharge. However, it will also reduce the $Q$ factor and the shunt impedance, and that will call for additional research and development of ceramic materials with reasonably high dielectric permittivity $\left(\varepsilon_{r}>20\right)$ and low loss tangent $\left(\tan \delta<10^{-4}\right)$. If $\mathrm{Ba}\left(\mathrm{Mg}_{1 / 3} \mathrm{Ta}_{2 / 3}\right) \mathrm{O}_{3}-\mathrm{Ba}\left(\mathrm{Mg}_{1 / 2} \mathrm{~W}_{1 / 2}\right) \mathrm{O}_{3}$ ceramics [48] could be used for dielectric cells, a DAA structure with much higher shunt impedance than what has been attained with the prototype described in this paper can be realized. Therefore, by using such a ceramic material and surface coating technology for dielectric cells, a new DAA structure which has high shunt impedance as high as that of this prototype and can deal with a problem of multipactor may be realized. While deepening our understanding of new ceramic materials and coating technologies and so on, we would like to work toward practical use of the DAA structure.

\section{CONCLUSIONS}

This paper presented the design, construction and cold testing of a prototype of five-cell C-band DAA structure performed at KEK. The DAA structure is a higher-order $\mathrm{TM}_{02 n}$ mode standing-wave accelerating structure and consists of ultralow-loss dielectric cells periodically arranged in a metallic enclosure. The advantage of the DAA structure is that it has an extremely high quality factor and a very high shunt impedance at room temperature. The design of the prototype DAA structure was optimized by the down-hill simplex algorithm to attain a high shunt impedance. As a result, the $Q_{0}$ and $Z_{\mathrm{sh}}$ of the designed prototype were estimated by the simulation to be more than 120,000 and $600 \mathrm{M} \Omega / \mathrm{m}$, respectively. The resonant frequency of the DAA structure was tuned by machining a concentric circular groove on the surface of the end copper plates.

A prototype of DAA structure was subsequently built in order to validate the electromagnetic characteristics and the frequency tuning scheme. Three types of dielectric cells ("regular," "end," and "hybrid" dielectric cells) are fabricated by sintering high-purity magnesia. The prototype was assembled by stacking these cells in the hollow copper cylinder, where was closed by end copper plates. 
The rf input coupler was designed and fabricated to be overcoupled considering the premeasurement results, while compensating for the frequency shifts in manufactured cells. Finally, the resonant frequency of the accelerating mode was tuned to the $\mathrm{C}$-band $(5.712 \mathrm{GHz})$ by machining a concentric circular groove on the surface of the two end plates. The unloaded quality factor of the accelerating mode was measured at 119,314 , which was within 5 percent of the designed values. A bead pull measurement of the prototype using the metallic beads moving along the beam axis showed good agreement with the simulated longitudinal electric field profile. Shunt impedance per unit length of the prototype was also estimated from the experimental results of the bead pull measurement as $Z_{\mathrm{sh}}=617 \mathrm{M} \Omega / \mathrm{m}$, which was within 2 percent of the simulated values. Further optimizations of the DAA structure to improve the shunt impedance and investigations into the damping scheme of higher and lower order modes for high current beam applications are under way.

Going forwards, the resonant frequency of accelerating mode will be more accurately tuned to the desired frequency considering the frequency shift due to evacuation and temperature variation in the DAA structure for a high power testing. The high power test of the DAA structure, currently in progress at KEK, will be reported in a separate publication.

\section{ACKNOWLEDGMENTS}

This work has been supported by Grant in Aid for JSPS Fellows Grant No. 13J08681. The authors would like to thank S. Miura and N. Shigeoka from the Mitsubishi Heavy Industries Mechatronics Systems Ltd. for their continued support of the development of DAA structures. The authors would also like to thank N. Toge and T. Natsui for helpful advice.

\section{APPENDIX A: DESIGN PROCEDURE OF THE PROTOTYPE}

This Appendix describes the procedure followed in determining the geometrical parameters of the cells that comprise a standing-wave five-cell DAA test structure to operate at the C-band frequencies.

A regular cell, as illustrated in Figs. 1(a) and 1(b), consists of dielectric cylinders with the inner radius $a_{1}$, the outer radius $b_{1}$ and dielectric disks with a thickness $D$. The beam hole irises have a diameter of $h$ and are arranged at a constant interval $L$. The cells are contained in a cylindrical metallic waveguide of the radius $c_{1}$. The end cell consists of dielectric disks with a thickness $D$ and dielectric coaxial dual cylinders with the length $l_{1}$. The inner and outer radii of the first and second cylinders are $h, r_{1}, a_{1}$ and $b_{1}$, respectively.

The eight geometrical parameters above are determined in the following manner. First, the phase shift of $\pi$ per cell

was chosen for the accelerating structure. The requirements from the energy of an injected beam and operating frequency determined the interval between adjacent dielectric disks, $L=26.24 \mathrm{~mm}$ :

$$
\frac{\omega L}{c}=\pi,
$$

where $\omega=2 \pi f_{0}, f_{0}=5.712 \mathrm{GHz}$ is the operating frequency, and $c$ is the speed of light. Considering the effective reflection of electromagnetic wave from the dielectric disks, the thickness of dielectric disk $D$ was chosen as being a quarter of the resonant wavelength in this dielectric material. The iris diameter, $h=10.00 \mathrm{~mm}$, was chosen for compatibility with the size of the electron beam available at a test bench of the KEK linac. The remaining five geometric parameters $\left(l_{1}, r_{1}, a_{1}, b_{1}, c_{1}\right)$ were optimized by using a down-hill simplex algorithm [36]. An evaluation function in this algorithm was defined to search an optimized model whose shunt impedance might become maximized. The eigenmode of the accelerating mode and the accelerator parameters (e.g., unloaded quality factor $Q_{0}$, shunt impedance per unit length $Z_{\mathrm{sh}}$ ) were calculated by the SUPERFISH code.

The optimization method of the DAA structure is as follows. Reference [34] has shown that the resonant frequency $f_{0}$ is sensitive to $b_{1}$. Therefore, the four geometrical parameters $\left(l_{1}, r_{1}, a_{1}, c_{1}\right)$, except for $b_{1}$, were changed in a

(a)

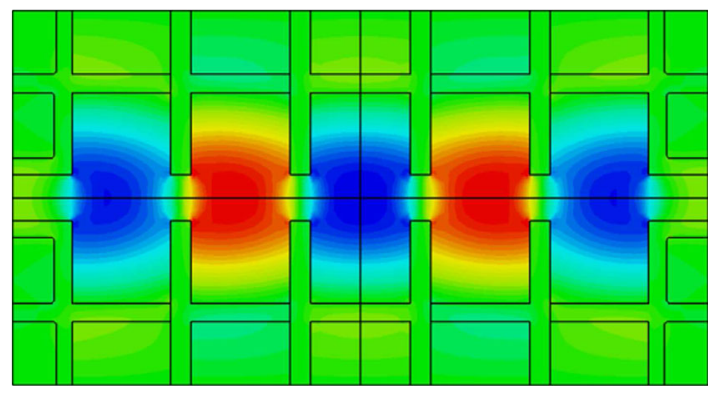

(b)

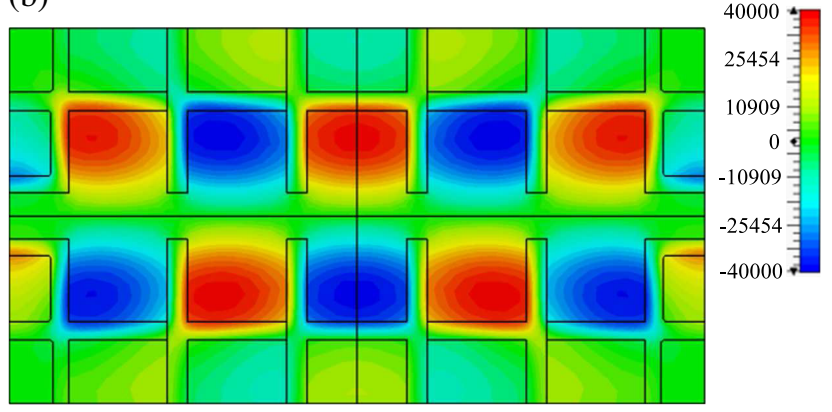

FIG. 14. Magnitude of (a) the longitudinal electric field distribution and (b) the rotating magnetic field distribution in the optimized DAA structure. The longitudinal cross section of the DAA structure is shown. This field intensity is shown in arbitrary unit. 
down-hill simplex algorithm and the resonant frequency of the prototype was tuned to the desired $f_{0}$ by adjusting the $b_{1}$ on each simplex point. Then the evaluation value was calculated based on the accelerator parameters. This process was repeated until a relative change ratio of the smallest evaluation value became less than $10^{-5}$.

The computed accelerator characteristics of the designed DAA structure, including the unloaded quality factor, $Q_{0}$, and the shunt impedance per unit length, $Z_{\mathrm{sh}}$ are shown in Table I. Figures 14(a) and 14(b) show the electric and magnetic field magnitude of the accelerating mode in the resulting optimized DAA structure, respectively.

\section{APPENDIX B: THE TOLERANCE OF GEOMETRICAL PARAMETERS}

This Appendix gives a short discussion on the tolerances of their geometrical parameters of dielectric structure cells.
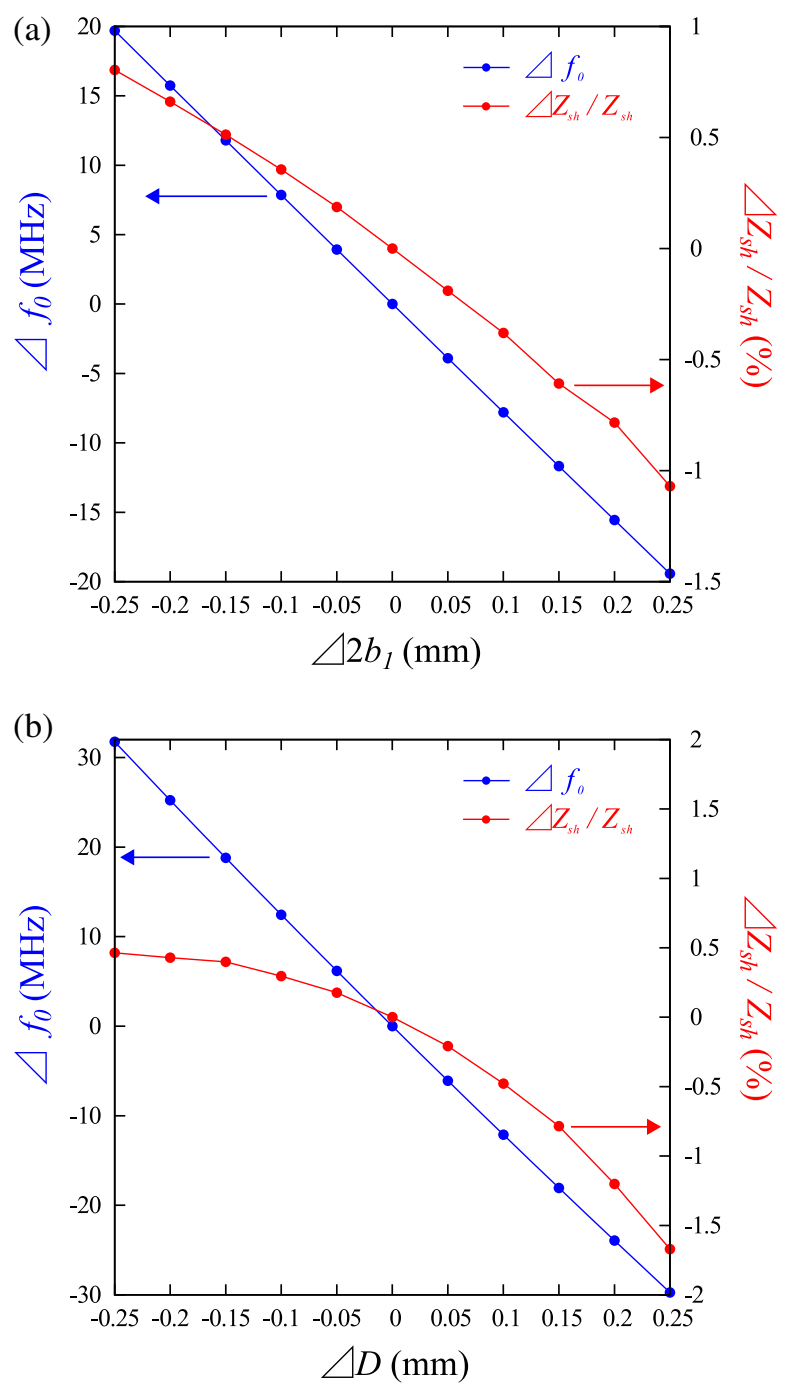

FIG. 15. Dependence of $\Delta f_{0}$ and $\Delta Z_{\text {sh }} / Z_{\text {sh }}$ on (a) the deviation of the outer radius $b_{1}$ at the dielectric cylinder, and on (b) the deviation of the thickness $D$ at the dielectric disk.
The tolerances were studied by calculating the dependence of $f_{0}$ and $Z_{\mathrm{sh}}$ on the geometrical parameters by using the SUPERFISH code. There, the deviations of resonance characteristics caused by changes of a certain geometrical parameter from $x$ to $x+d x$ were evaluated. It was found that the resonant frequency of the prototype is especially sensitive to $b_{1}$ and $D$. Figures 15 (a) and 15(b) show the amount of shifts of the resonant frequency $\left(\Delta f_{0}\right)$ and shunt impedance $\left(\Delta Z_{\mathrm{sh}}\right)$ as functions of the outer radius $b_{1}$ of dielectric cylinders and the thickness $D$ at the dielectric disks, respectively. Figure 15 indicates that a deviation of $0.15 \mathrm{~mm}$ in either $2 b_{1}$ or $D$ causes frequency shifts of more than $10 \mathrm{MHz}$. Therefore, in building our prototype we set the fabrication errors of $2 b_{1}$ and $D$ to be $-0.1 \mathrm{~mm} /+0 \mathrm{~mm}$ and $-0.05 \mathrm{~mm} /+0 \mathrm{~mm}$, respectively. This is so the target value of the resonant frequency $(5.712 \mathrm{GHz})$ can be attained by tuning the groove depths on end cells as discussed in the main text.

[1] R. C. Sethi, Electron beam accelerators for materials processing: a barc scenatrio, in Proceedings of the APAC 2004, Gyeongju, Korea (JACoW, Geneva, 2004), pp. 708-710.

[2] L. Auditore, R. C. Barna, D. DePasquale, A. Italiano, A. Trifiro, and M. Trimarchi, Pulsed $5 \mathrm{MeV}$ standing wave electron linac for radiation processing, Phys. Rev. ST Accel. Beams 7, 030101 (2004).

[3] E. Tanabe, Y. Fineberg, H. Matsumoto, and T. Shintake, Medical applications of c-band accelerator technologies, in Proceedings of the 1998 Linear Accelerator Conference, Argonne, IL, USA (JACoW, Geneva, 1998), pp. 627-629.

[4] J. Hyun, M. Yoshida, M. Satoh, T. Sakai, Y. Hayakawa, T. Tanaka, K. Hayakawa, I. Sato, and K. Endo, in Proceedings of IPAC2015, Richmond, VA, USA (JACoW, Geneva, 2015), pp. 1604-1606.

[5] T. Shintake et al., A compact free-electron laser for generating coherent radiation in the extreme ultraviolet region, Nat. Photonics 2, 555 (2008).

[6] T. Behnke, J. E. Brau, B. Foster, J. Fuster, M. Harrison, J. M. Paterson, M. Peskin, M. Stanitzki, N. Walker, and H. Yamamoto, The International Linear Collider Technical Design Report-Volume 1: Executive Summary (2013), http://www.linearcollider.org/ILC/Publications/TechnicalDesign-Report.

[7] M. Aicheler, P. Burrows, M. Draper, T. Garvey, P. Lebrun, K. Peach, N. Phinney, H. Schmickler, D. Schulte, and N. Toge, CLIC Conceptual Design Report No. CERN-2012007, 2012.

[8] S. Dobert, A. Grudiev, G. Riddone, W. Wuensch, R. Zennaro, T. Higo, S. Matsumoto, K. Yokoyama, C. Adolphsen, F. Wang, and J. Wang, in Proceedings of the 25th International Linear Accelerator Conference, LINAC-2010, Tsukuba, Japan (KEK, Tsukuba, Japan, 2010), pp. 208-210.

[9] T. Higo, in, Proceedings of the 25th International Linear Accelerator Conference, LINAC-2010, Tsukuba, Japan (KEK, Tsukuba, Japan, 2010), pp. 1038-1042. 
[10] V. A. Dolgashev, S. G. Tantawi, A. D. Yeremian, Z. Li, Y. Higashi, and B. Spataro, in Proceedings of IPAC2011, San Sebastian, Spain (EPS-AG, Spain, 2011), pp. 241-243.

[11] M. DalForno, V. Dolgashev, G. Bowden, C. Clarke, M. Hogan, D. McCormick, A. Novokhatski, B. Spataro, S. Weathersby, and S. G. Tantawi, rf breakdown tests of mm-wave metallic accelerating structures, Phys. Rev. Accel. Beams 19, 011301 (2016).

[12] W. Gai, R. Konecny, and J. Simpson, in Proceedings of the 1997 Particle Accelerator Conference, Vancouver, Canada (IEEE, Piscataway, NJ, 1998), pp. 636-638.

[13] P. Zou, W. Gai, R. Konecny, X. Sun, T. Wong, and A. Kanareykin, Construction and testing of an $11.4 \mathrm{GHz}$ dielectric structure based traveling wave accelerator, Rev. Sci. Instrum. 71, 2301 (2000).

[14] W. Liu, C. Jing, W. Gai, R. Konecny, and J. G. Power, in Proceedings of the 2003 Particle Accelerator Conference, Portland, Oregon, USA (IEEE, Piscataway, NJ, 2003), pp. 1810-1812.

[15] E. Chojnacki, W. Gai, C. Ho, R. Konecny, S. Mtingwa, J. Norem, M. Rosing, P. Schoessow, and J. Simpson, Measurement of deflection-mode damping in an accelerating structure, J. Appl. Phys. 69, 6257 (1991).

[16] S. Frankel, $T M_{0,1}$ mode in circular wave guides with two coaxial dielectrics, J. Appl. Phys. 18, 650 (1947).

[17] G. G. Bruck and E. R. Wicher, Slow transverse magnetic waves in cylindrical guides, J. Appl. Phys. 18, 766 (1947).

[18] A. A. Oliner, Remarks on slow waves in cylindrical guides, J. Appl. Phys. 19, 109 (1948).

[19] T.-B. Zhang, J. L. Hirshfield, T. C. Marshall, and B. Hafizi, Stimulated dielectric wake-field accelerator, Phys. Rev. E 56, 4647 (1997).

[20] K. DeckerFrench, Development of a dielectric loaded RF cavity for a muon accelerator, Nucl. Instrum. Methods Phys. Res., Sect. A 624, 731 (2010).

[21] C. Jing, A. Kanareykin, S. Kazakov, W. Liu, E. Nenasheva, P. Schoessow, and W. Gai, Development of a dual-layered dielectric-loaded accelerating structure, Nucl. Instrum. Methods Phys. Res., Sect. A 594, 132 (2008).

[22] P. Zou, L. Xiao, X. Sun, and W. Gai, in Proceedings of the 2001 Particle Accelerator Conference, Chicago (IEEE, Piscataway, NJ, 2001), pp. 3966-3968.

[23] A. V. Smirnov and E. Savin, Hybrid metal-dielectric, slow wave structure with magnetic coupling and compensation, Nucl. Instrum. Methods Phys. Res., Sect. A 820, 48 (2016).

[24] A. V. Smirnov, S. Boucher, S. Kutsaev, J. Hartzell, and E. Savin, Multi-cell disk-and-ring tapered structure for compact RF linacs, Nucl. Instrum. Methods Phys. Res., Sect. A 830, 294 (2016).

[25] J. X. Zhang, B. J. Munroe, H. Xu, M. A. Shapiro, and R. J. Temkin, High power experimental studies of hybrid photonic band gap accelerator structures, Phys. Rev. Accel. Beams 19, 081304 (2016).

[26] C. Jing, W. M. Liu, W. Gai, J. G. Power, and T. Wong, Mode analysis of a multilayered dielectric-loaded accelerating structure, Nucl. Instrum. Methods Phys. Res., Sect. A 539, 445 (2005).
[27] C. Jing, W. Liu, W. Gai, J. G. Power, and A. Kanareykin, in Proceedings of the 2005 Particle Accelerator Conference, Knoxville, Tennessee (IEEE, Piscataway, NJ, 2005), pp. 1592-1594.

[28] E. S. Kim and C. J. Jeon, Microwave dielectric properties of $\mathrm{ATiO}_{3}(\mathrm{~A}=\mathrm{Ni}, \mathrm{Mg}, \mathrm{Co}, \mathrm{Mn})$ ceramics, J. Eur. Ceram. Soc. 30, 341 (2010).

[29] T. Tsunookaa, M. Androua, Y. Higashidaa, H. Sugiurab, and $\mathrm{H}$. Ohsatoc, Effects of $\mathrm{TiO}_{2}$ on sinterability and dielectric properties of high-Q forsterite ceramics, J. Eur. Ceram. Soc. 23, 2573 (2003).

[30] H. Ohsato, T. Tsunooka, T. Sugiyama, K. Kakimoto, and H. Ogawa, Forsterite ceramics for millimeterwave dielectrics, J. Electroceram. 17, 445 (2006).

[31] N. M. Alford and S. J. Penn, Sintered alumina with low dielectric loss, J. Appl. Phys. 80, 5895 (1996).

[32] C. L. Huang, J. J. Wang, and C. Y. Huang, Sintering behavior and microwave dielectric properties of nano alpha-alumina, Mater. Lett. 59, 3746 (2005).

[33] C. L. Huang, J. J. Wang, and C. Y. Huang, Microwave dielectric properties of sintered alumina using nano-scaled powders of $\alpha$ alumina and $\mathrm{TiO}_{2}$, J. Am. Ceram. Soc. 90, 1487 (2007).

[34] D. Satoh, M. Yoshida, and N. Hayashizaki, Dielectric assist accelerating structure, Phys. Rev. Accel. Beams 19, 011302 (2016).

[35] D. Satoh, Doctoral thesis, Tokyo Institute of Technology (unpublished).

[36] J. A. Nelder and R. Mead, A simplex method for function minimization, Computer Journal (UK) 7, 308 (1965).

[37] E. L. Ginzton, Microwave Measurements (McGraw-Hill Book Company, Inc., New York, 1957).

[38] Y. Taniguchi, F. Sakamoto, T. Natsui, T. Yamamoto, E. Hashimoto, K. W. Lee, M. Uesaka, M. Yoshida, T. Higo, S. Fukuda, and M. Akemoto, Upgrade of X-band thermionic cathode RF gun for Compton scattering X-ray source, Nucl. Instrum. Methods Phys. Res., Sect. A 608, S113 (2009).

[39] J. C. Slater, Microwave Electronics (D. Van Nostrand Co., Inc., New York, 1950).

[40] J. H. Billen and L. M. Young, Los Alamos National Laboratory Report No. LA-UR-96-1834, 1996.

[41] L. C. Maier Jr. and J.C. Slater, Field strength measurements in resonant cavities, J. Appl. Phys. 23, 68 (1952).

[42] D. A. Hill, Electromagnetic Fields in Cavities: Deterministic and Statistical Theories, Vol. 35 (John Wiley \& Sons, Hoboken, NJ, 2009).

[43] H. Hahn, R. Calaga, P. Jain, E. C. Johnson, and W. Xu, HOM identification by bead pulling in the Brookhaven ERL cavity, Nucl. Instrum. Methods Phys. Res., Sect. A 734, 72 (2014).

[44] C. Jing, A. Kanareykin, R. Konecny, J. G. Power, S. H. Gold, and S. Kazakov, in Proceedings of the 2007 Particle Accelerator Conference, Albuquerque, New Mexico, USA (IEEE, Piscataway, NJ, 2007), pp. 3151-3153.

[45] J. G. Power, W. Gai, S. H. Gold, A. K. Kinkead, R. Konecny, C. Jing, W. Liu, and Z. Yusof, Observation of Multipactor in an Alumina-Based Dielectric-Loaded Accelerating Structure, Phys. Rev. Lett. 92, 164801 (2004). 
[46] C. Jing, J. G. Power, R. Konecny, W. Gai, S. H. Gold, and A. K. Kinkead, in Proceedings of the 2005 Particle Accelerator Conference, Knoxville, Tennessee (Ref. [27), pp. 1566-1568.

[47] W. Gai, P. Schoessow, B. Cole, R. Konecny, J. Norem, J. Rosenzweig, and J. Simpson, Experimental Demonstration of Wake-Field Effects in Dielectric Structures, Phys. Rev. Lett. 61, 2756 (1988).

[48] M. Furuta and A. Ochi, Microwave Dielectric Properties for $\mathrm{Ba}\left(\mathrm{Mg}_{1 / 3} \mathrm{Ta}_{2 / 3}\right) \mathrm{O}_{3}-A\left(M g_{1 / 2} W_{1 / 2}\right) O_{3}(A=B a, S r$, and $\mathrm{Ca}$ ) Ceramics, Jpn. J. Appl. Phys. 33, 5482 (1994). 